Draft VERSION June 8, 2017

Typeset using $\mathrm{IAT}_{\mathrm{E} X}$ modern style in AASTeX61

\title{
ON THE AGE OF THE TRAPPIST-1 SYSTEM
}

\author{
AdAm J. Burgasser ${ }^{1}$ AND ERIC E. MAMAJEK ${ }^{2,3}$ \\ ${ }^{1}$ Department of Physics, University of California, San Diego, CA 92093, USA \\ ${ }^{2}$ Jet Propulsion Laboratory, California Institute of Technology, 4800 Oak Grove Drive, Pasadena, \\ CA 91109, USA \\ ${ }^{3}$ Department of Physics 8 Astronomy, University of Rochester, Rochester, NY 14627, USA \\ (Received 1 June 2017; Revised TBD; Accepted TBD) \\ Submitted to ApJ
}

\begin{abstract}
The nearby ( $\mathrm{d}=12$ pc) M8 dwarf star TRAPPIST-1 (2MASS J23062928-0502285) hosts a compact system of at least seven exoplanets with sizes similar to Earth. Given its importance for testing planet formation and evolution theories, and for assessing the prospects for habitability among Earth-size exoplanets orbiting the most common type of star in the Galaxy, we present a comprehensive assessment of the age of this system. We collate empirical age constraints based on the color-absolute magnitude diagram, average density, lithium absorption, surface gravity features, metallicity, kinematics, rotation, and magnetic activity; and conclude that TRAPPIST-1 is a transitional thin/thick disk star with an age of $7.6 \pm 2.2$ Gyr. The star's colormagnitude position is consistent with it being slightly metal-rich $([\mathrm{Fe} / \mathrm{H}] \simeq+0.06)$, in line with its previously reported near-infrared spectroscopic metallicity; and it has a radius $\left(R=0.121 \pm 0.003 R_{\odot}\right)$ that is larger by $8-14 \%$ compared to solar-metallicity evolutionary models. We discuss some implications of the old age of this system with regard to the stability and habitability of its planets.
\end{abstract}

Keywords: stars: activity — stars: atmospheres — stars: low-mass stars: individual (2MASS J23062928-0502285, TRAPPIST1) 


\section{INTRODUCTION}

TRAPPIST-1 (2MASS J23062928-0502285; Gizis et al. 2000) is as an ultracool M8 dwarf $12 \mathrm{pc}$ from the Sun which was recently identified to host at least seven Earth-sized planets, three orbiting within the star's habitable zone (Gillon et al. 2016, 2017; Luger et al. 2017). The planets were identified by both ground-based and space-based transit observations, and span orbit periods of 1.5-19 days and orbital semi-major axes of 0.011-0.062 AU (21-114 stellar radii). These observations and others have provided important constraints on the physical parameters of the star itself, summarized in Table 1.

One crucial parameter of TRAPPIST-1 that is poorly constrained by observations to date is its age, due to the weak empirical age diagnostics currently available for ultracool M dwarfs. While rotation (gryochronology), activity diagnostics, and lithium depletion are standard age-dating tools for solar-type stars (Soderblom 2010), the physical properties of ultracool dwarfs restrict the application of these tools at the bottom of the main sequence. The low ionization of ultracool dwarf photospheres reduces their coupling with magnetic winds, resulting in spin-down timescales that can exceed the age of the Milky Way Galaxy (West et al. 2008). Depletion of lithium, which provide approximate ages for solar-type stars up to 1-2 Gyr (Sestito \& Randich 2005), is complete for fully-convective low-mass stars by $\sim 200 \mathrm{Myr}$, and so is useful only for young ultracool dwarfs (Stauffer et al. 1998). Spectral age diagnostics, such as surface gravity sensitive features, are also limited to ages older than $\sim 300 \mathrm{Myr}$ (Allers \& Liu 2013). While the kinematics of TRAPPIST-1 suggest it to be an "old disk" star (Leggett 1992; Burgasser et al. 2015), such labels are insufficient to firmly constain an age. Filippazzo et al. (2015) report an fairly unconstrained age constraint of 0.5-10 Gyr; while Luger et al. (2017) adopt a more constrained, but still broad, range of 3-8 Gyr. In contrast, several studies in the literature have argued that TRAPPIST-1 must be young based on the strength of its nonthermal magnetic emission (Bourrier et al. 2017; O’Malley-James \& Kaltenegger 2017).

Age is necessary for understanding the formation, orbital evolution, stability, and surface evolution (including habitability) of the planets orbiting TRAPPIST-1. With this in mind, we present an analysis of various age-related empirical diagnostics for this source that allow us to more precisely quantify its age. In Section 2 we analyze in turn the color-absolute magnitude diagram, average density, lithium abundance, surface gravity features, metallicity, kinematics, rotation, and magnetic activity as age diagnostics for the star. In Section 3 we summarize these into a concordance age of $7.6 \pm 2.2 \mathrm{Gyr}$, and discuss implications on the stability and habitability of the planetary system. 
Table 1. Stellar Parameters for TRAPPIST-1

\begin{tabular}{|c|c|c|c|}
\hline Parameter & Value & Units & Ref. \\
\hline \multicolumn{4}{|c|}{ Physical Parameters } \\
\hline $\log _{10} \mathrm{~L} / \mathrm{L}_{\mathrm{bol}}$ & $-3.28 \pm 0.03$ & $\operatorname{dex}$ & 1 \\
\hline $\mathrm{T}_{\mathrm{eff}}$ & $2560 \pm 50$ & $\mathrm{~K}$ & 2 \\
\hline$[\mathrm{Fe} / \mathrm{H}]$ & $+0.04 \pm 0.08$ & $\operatorname{dex}$ & 2 \\
\hline$P_{\text {rot }}$ & $3.295 \pm 0.003$ & day & 3 \\
\hline$v \sin i$ & 6 & $\mathrm{~km} \mathrm{~s}^{-1}$ & 4 \\
\hline $\log _{10}\left(\mathrm{~L}_{H \alpha} / \mathrm{L}_{b o l}\right)$ & -4.85 to -4.60 & $\operatorname{dex}$ & $5-8$ \\
\hline $\log _{10}\left(\mathrm{~L}_{X} / \mathrm{L}_{b o l}\right)$ & $-3.52 \pm 0.17$ & $\operatorname{dex}$ & 9 \\
\hline Radius & $0.117 \pm 0.004$ & $\mathrm{R}_{\odot}$ & 2 \\
\hline$\ldots$ & $0.121 \pm 0.003$ & $\mathrm{R}_{\odot}$ & 10 \\
\hline Mass & $0.080 \pm 0.007$ & $\mathrm{M}_{\odot}$ & 2 \\
\hline Density & $50.7_{-2.2}^{+1.2}$ & $\rho_{\odot}$ & 2 \\
\hline Age & $7.6 \pm 2.2$ & Gyr & 10 \\
\hline \multicolumn{4}{|c|}{ Astrometric/Kinematic Parameters } \\
\hline$\alpha$ & 346.6250957 & deg & 11 \\
\hline$\delta$ & -5.0428081 & $\operatorname{deg}$ & 11 \\
\hline$\mu_{\alpha}$ & $922.0 \pm 0.6$ & mas yr ${ }^{-1}$ & 12 \\
\hline$\mu_{\delta}$ & $-471.9 \pm 0.9$ & $\operatorname{mas} \mathrm{yr}^{-1}$ & 12 \\
\hline$\pi$ & $80.09 \pm 1.17$ & mas & 12 \\
\hline Distance & $12.49 \pm 0.18$ & pc & 12 \\
\hline$v_{r}$ & $-51.688 \pm 0.014$ & $\mathrm{~km} \mathrm{~s}^{-1}$ & 4 \\
\hline$U$ & $-43.8 \pm 0.7$ & $\mathrm{~km} \mathrm{~s}^{-1}$ & 10 \\
\hline$V$ & $-66.3 \pm 0.5$ & $\mathrm{~km} \mathrm{~s}^{-1}$ & 10 \\
\hline$W$ & $11.0 \pm 0.3$ & $\mathrm{~km} \mathrm{~s}^{-1}$ & 10 \\
\hline$S$ & $80.0 \pm 0.7$ & $\mathrm{~km} \mathrm{~s}^{-1}$ & 10 \\
\hline \multicolumn{4}{|c|}{ Photometric Parameters } \\
\hline$V$ & $18.75 \pm 0.03$ & mag & 13 \\
\hline$R_{C}$ & $16.401 \pm 0.004$ & mag & 14 \\
\hline$I_{C}$ & $13.966 \pm 0.002$ & mag & 14 \\
\hline$J$ & $11.35 \pm 0.02$ & mag & 15 \\
\hline$H$ & $10.72 \pm 0.02$ & mag & 15 \\
\hline$K_{s}$ & $10.30 \pm 0.02$ & mag & 15 \\
\hline$W 1$ & $10.07 \pm 0.02$ & mag & 11 \\
\hline
\end{tabular}

Table 1 continued on next page 
Burgasser \& MAMAJEK

Table 1 (continued)

\begin{tabular}{lccl}
\hline \multicolumn{1}{c}{ Parameter } & Value & Units & Ref. \\
\hline$W 2$ & $9.81 \pm 0.02$ & $\mathrm{mag}$ & 11 \\
$W 3$ & $9.51 \pm 0.04$ & $\mathrm{mag}$ & 11 \\
$V-K_{s}$ & $8.45 \pm 0.04$ & $\mathrm{mag}$ & 13,15 \\
$M_{V}$ & $18.27 \pm 0.04$ & $\mathrm{mag}$ & 10 \\
$M_{J}$ & $10.87 \pm 0.04$ & $\mathrm{mag}$ & 10 \\
$M_{K_{s}}$ & $9.81 \pm 0.04$ & $\mathrm{mag}$ & 10 \\
\hline
\end{tabular}

References- (1) Filippazzo et al. (2015); (2) Gillon et al. (2016); (3) Vida et al. (2017); (4) Barnes et al. (2014); (5) Gizis et al. (2000) (6) Reiners \& Basri (2008) (7) Barnes et al. (2014); (8) Burgasser et al. (2015); (9) Wheatley et al. (2017) (10) This paper; (11) AllWISE epoch 2010.5589 (Cutri et al. 2013); (12) Weinberger et al. (2016); (13) Winters et al. (2015); (14) Liebert \& Gizis (2006); (15) 2MASS (Skrutskie et al. 2006);

\section{ANALYSIS}

\subsection{Age Constraints from the HR Diagram}

The locations of TRAPPIST-1 and other late M-type spectral standards and field stars on the $M_{K s}$ versus $\left(V-K_{s}\right)$ color-absolute magnitude diagram (CMD) are shown in Figure 1. The comparison data are drawn from Dieterich et al. (2014) and Winters et al. (2015) for nearby $\mathrm{M}$ dwarfs with trigonometric parallaxes. A polynomial fit to these data between $3.5<\left(V-K_{s}\right)<11.8$ yields:

$$
\begin{array}{r}
M_{K s}=26.987-20.6315\left(V-K_{s}\right)+6.88044\left(V-K_{s}\right)^{2}-1.01665\left(V-K_{s}\right)^{3} \\
+0.0707374\left(V-K_{s}\right)^{4}-0.00188517\left(V-K_{s}\right)^{5}
\end{array}
$$

The rms scatter among field $M$ dwarfs is 0.48 mag. Recent spectroscopic surveys of field $\mathrm{M}$ dwarfs find median metallicities ranging from $[\mathrm{Fe} / \mathrm{H}] \simeq+0.04$ (Newton et al. 2014) to $[\mathrm{Fe} / \mathrm{H}] \simeq-0.03$ (Mann et al. 2015), with $1 \sigma$ dispersions of $\sim 0.2$ dex. Hence, this CMD sequence is largely representative of a solar metallicity population.

Also shown on the CMD are recent isochrones of low mass stars of solar composition from Baraffe et al. (2015) for ages of 0.2, 0.3, 0.5, 3, and 8 Gyr and masses of 0.07, 0.08 , and $0.09 \mathrm{M}_{\odot}$, spanning the mass estimates for TRAPPIST-1. These isochrones reproduce the empirical locus for late-M dwarfs to an accuracy of $\sim 0.1-0.2 \mathrm{mag}$ in $\mathrm{M}_{K s}$ or $\sim 0.2 \mathrm{mag}$ in $\left(V-K_{s}\right)$ color; but not the greater spread, which is likely due to metallicity scatter (López-Morales 2007). Binaries are relatively rare among late M dwarfs ( 10-20\%; Allen 2007; Kraus \& Hillenbrand 2012) and most field stars will be older than the pre-main-sequence contraction timescale of hundreds of Myr. 
TRAPPIST-1 lies $0.15 \pm 0.04$ mag above (brighter than) the empirical locus. This offset could be interpretted as TRAPPIST-1 being a $0.2-0.3$ Gyr-old, $0.07 \mathrm{M}_{\odot}$ brown dwarf. However, this interpretation would predict a density of $38 \rho_{\odot}$ based on the Baraffe et al. (2015) models, which is much lower than the measured density from transit observations (see below). We can also rule out contamination from a stellar or substellar companion based on previous adaptive optics surveys (Siegler et al. 2003; Bouy et al. 2003; Gizis et al. 2003; Janson et al. 2012) and multi-epoch radial velocity surveys (Tanner et al. 2012; Barnes et al. 2014).

Instead, the magnitude offset of TRAPPIST-1 is likely due to a slightly super-solar metallicity. The offset is similar to that of the M8 V standard VB 10 (aka GJ 752B), and it and its $\mathrm{M} 3 \mathrm{~V}$ companion GJ 752A both have $[\mathrm{Fe} / \mathrm{H}] \simeq 0.1$ (Newton et al. 2014; Mann et al. 2015), consistent with the spectroscopic value for TRAPPIST-1 (Gillon et al. 2016). Alternately, attributing the 0.48 mag scatter in absolute magnitude for the CMD empirical locus entirely to metallicity scatter $(\sim 0.2$ dex $)$ would imply a metallicity-absolute magnitude gradient of $\Delta[\mathrm{Fe} / \mathrm{H}] / \Delta \mathrm{M}_{K s} \simeq-0.4 \mathrm{dex} \mathrm{mag}^{-1}$, with metal-rich stars being brighter. Under this interpretation, the -0.15 mag absolute magnitude offset for TRAPPIST-1 would be consistent with $[\mathrm{Fe} / \mathrm{H}]=+0.06 \mathrm{dex}$, again consistent with the spectroscopic metallicity.

Unfortunately, currently-available evolutionary models for the very lowest mass stars and brown dwarfs are defined only for solar metallicities, preventing us from disentangling age and metallicity effects. Our CMD analysis therefore reinforces a supersolar metallicity for TRAPPIST-1, but cannot constrain its age.

\subsection{Age Constraints from Stellar Density}

Average stellar density, an observable from transit lightcurve analysis (Seager \& Mallén-Ornelas 2003), provides an independent check on the evolutionary state of TRAPPIST-1. Figure 1 shows the predicted stellar densities for stars and brown dwarfs from the solar-metallicity evolutionary models of Burrows et al. (1997, 2001) and Baraffe et al. (2015) as a function of age, constrained to have the observed luminosity of TRAPPIST-1. At this luminosity, young, contracting, substellar objects have densities that increase up to an age of approximately $500 \mathrm{Myr}$; beyond $1 \mathrm{Gyr}$ (corresponding to hydrogen-burning low mass stars), densities plateau. The Burrows et al. models predict densities $17 \%$ higher than the Baraffe et al. models, corresponding to radii that are $6 \%$ smaller. The average density of TRAPPIST-1 is on the rising portion of this trend, and intersects with the Burrows et al. and Baraffe et al. models at ages of $0.3 \mathrm{Gyr}$ and $0.4 \mathrm{Gyr}$, respectively, corresponding to masses at the hydrogen-burning mass limit $\left(0.071 \mathrm{M}_{\odot}\right.$ and $\left.0.079 \mathrm{M}_{\odot}\right)$.

These comparisons again suggest that TRAPPIST-1 is relatively young. However, two factors must be considered. First, there are the previously-noted metallicity effects. Among $-0.04<[\mathrm{Fe} / \mathrm{H}]<0.12$ low-mass stars, López-Morales (2007) found 

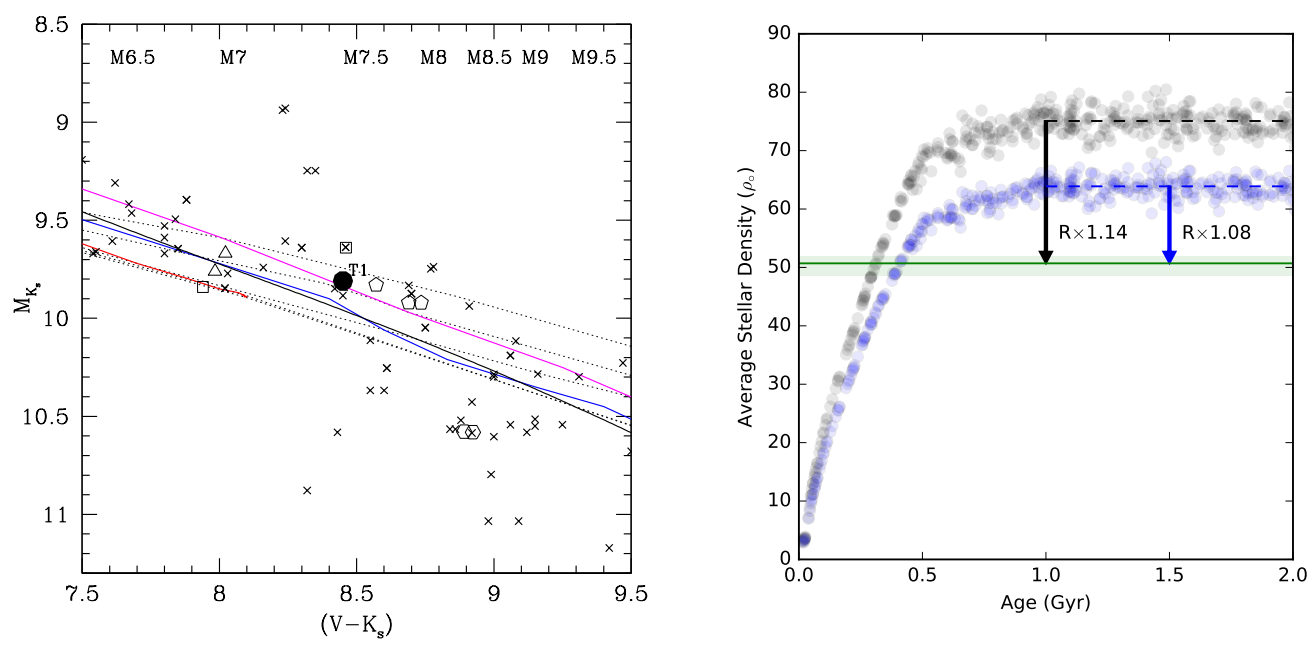

Figure 1. (Left): Color-absolute magnitude diagram $\left(M_{K s}\right.$ versus $\left.V-K_{s}\right)$ for TRAPPIST1, late-M spectral standard stars from Kirkpatrick et al. (1991, 1997, 2010) and Henry et al. (2002, 2004), and late-M field stars from Dieterich et al. (2014) and Winters et al. (2015). TRAPPIST-1 is plotted as a large filled circle; the spectral standards are indicated as open triangles $(\mathrm{M} 7 \mathrm{~V})$, open squares $(\mathrm{M} 7.5 \mathrm{~V})$, open pentagons $(\mathrm{M} 8 \mathrm{~V})$, and open hexagons $(\mathrm{M} 8.5 \mathrm{~V})$; all others as crosses. The color-magnitude locus for $\mathrm{M}$ dwarfs is plotted as a solid black line. Isochrones from Baraffe et al. (2015) for ages of 0.2, 0.3, 0.5, 3 and $8 \mathrm{Gyr}$ (dashed lines, older isochrones toward the bottom) and masses of 0.07, 0.08, and $0.09 \mathrm{M}_{\odot}$ (from top to bottom, solid magenta, blue, and red lines, respectively) are also shown. Given the slow evolution of $\mathrm{M}$ dwarfs on the main sequence, the 3 and $8 \mathrm{Gyr}$ isochrones are indistinguishable on the scale of the diagram. Along the top are listed the approximate spectral type based on $V-K_{s}$ color. (Right): Average stellar density in solar units as a function of age based on the theoretical models of Burrows et al. (1997, 2001, black symbols) and Baraffe et al. (2015, blue symbols) for a luminosity $\log _{10} \mathrm{~L} / \mathrm{L}_{\mathrm{bol}}=-3.28 \pm 0.03$. Values were selected by Monte Carlo sampling in age (uniform distribution) and luminosity (normal distribution). The average density measured for TRAPPIST-1 from Gillon et al. (2016), 50.7 $7_{-2.2}^{+1.2} \rho_{\odot}$, is indicated by the horizontal green line and region. The radius scale factors needed to "inflate" the models for ages $>1$ Gyr (dashed lines) to the average density of TRAPPIST-1 are indicated by the arrows.

evolutionary models underpredict stellar radii by 10-20\%, a correction factor sufficient to bring the density plateaus of the Baraffe et al. and Burrows et al. models in line with the observed density of TRAPPIST-1. Second, magnetic activity can also modulate the radii of low mass stars and brown dwarfs (López-Morales 2007; Reiners et al. 2007; Chabrier et al. 2007; MacDonald \& Mullan 2009; Mohanty et al. 2010). Empirical relations from Stassun et al. (2012) predict a modest effect: 0-4\% based on X-ray emission, 4-6\% based on $\mathrm{H} \alpha$ emission. However, theoretical models by Chabrier et al. (2007) show radii can range over $0.10-0.14 \mathrm{R}_{\odot}$ at $\mathrm{M}=0.08 \mathrm{M}_{\odot}$ for (black) spot coverage of up to $50 \%$, more than sufficient to cover the offset in TRAPPIST-1's stellar density. Kepler data have confirm the presence of cool, stable magnetic spots on TRAPPIST-1 (Luger et al. 2017; Vida et al. 2017), so these may play a role in radius inflation. 
Given that both metallicity and magnetic activity likely play a role in setting the average stellar density of TRAPPIST-1, the current unavailability of appropriate evolutionary models encapsulating these effects again prevents us from extracting meaningful age constraints from this physical parameter.

\subsection{Age Constraints from Lithium Depletion}

Multiple studies have reported the absence of $6708 \AA$ Li I absorption in the optical spectrum of TRAPPIST-1, indicating depletion of this element in its fully convective interior (Reiners \& Basri 2009; Burgasser et al. 2015). As noted above, theoretical models of solar-metallicity stars and brown dwarfs more massive than $0.06 \mathrm{M}_{\odot}$ show full depletion within 200 Myr (Bildsten et al. 1997; Burke et al. 2004). Similarly, the Burrows et al. $(1997,2001)$ and Baraffe et al. (2015) evolutionary models predict a minimum age of $190 \mathrm{Myr}$ for a $0.06 \mathrm{M}_{\odot}$ brown dwarf with the observed luminosity of TRAPPIST-1. These estimates provide a lower limit to TRAPPIST-1's age that is already incorporated into the age range proposed by Filippazzo et al. (2015).

\subsection{Age Constraints from Surface Gravity Features}

Low-resolution near-infrared spectra presented in Gillon et al. (2016) are generally consistent with the M8 dwarf spectral standard VB 10. Howeer, there are some notable peculiarities, including weaker $\mathrm{FeH}$ absorption and a more triangular $H$-band peak (Figure 2). Application of the Allers \& Liu (2013) surface gravity indices yields a gravity classification of INT-G for this source, suggesting a low surface gravity and young ( 100-300 Myr) age. Moreover, comparison of its near-infrared spectrum to the entirety of the SpeX Prism Library (Burgasser 2014) uncovers an excellent match to the M7 dwarf 2MASS J2352050-110043 (Cruz et al. 2007; hereafter 2MASS J2352-1100), a source identified by Gagné et al. (2015a) and Aller et al. (2016) as a possible kinematic member of the $\sim 110$ Myr AB Doradus association (Luhman et al. 2005; Barenfeld et al. 2013). These lines of evidence again suggest TRAPPIST-1 could be a young brown dwarf.

However, 2MASS J2352-1100 lacks Li I absorption, expected for a 110 Myr M8 dwarf; and it kinematic association with AB Doradus is based on proper motion and estimated distance alone, and may be spurious. TRAPPIST-1's kinematics firmly rule out membership in AB Doradus or any nearby young moving group (Malo et al. 2013; Gagné et al. 2014); and neither TRAPPIST-1 nor 2MASS J2352-1100 exhibit the enhanced VO absorption generally seen in the spectra of low-gravity M and L dwarfs (Kirkpatrick et al. 2008; Cruz et al. 2009; Allers \& Liu 2013). The nearinfrared spectrum of TRAPPIST-1 is also a good match to those of the M8 dwarfs LP 938-71 and 2MASS J2341286-113335 (Figure 2), neither of which are reported to be unusually young or active (Cruz et al. 2007; Schmidt et al. 2007).

We conclude that the INT-G gravity classifications for both sources are unrelated to youth, and may arise from other physical factors, as previously reported for the high velocity M6.5 2MASS J02530084+1652532 (aka Teegarden's star, $V_{t a n}=93 \mathrm{~km} \mathrm{~s}^{-1}$; 

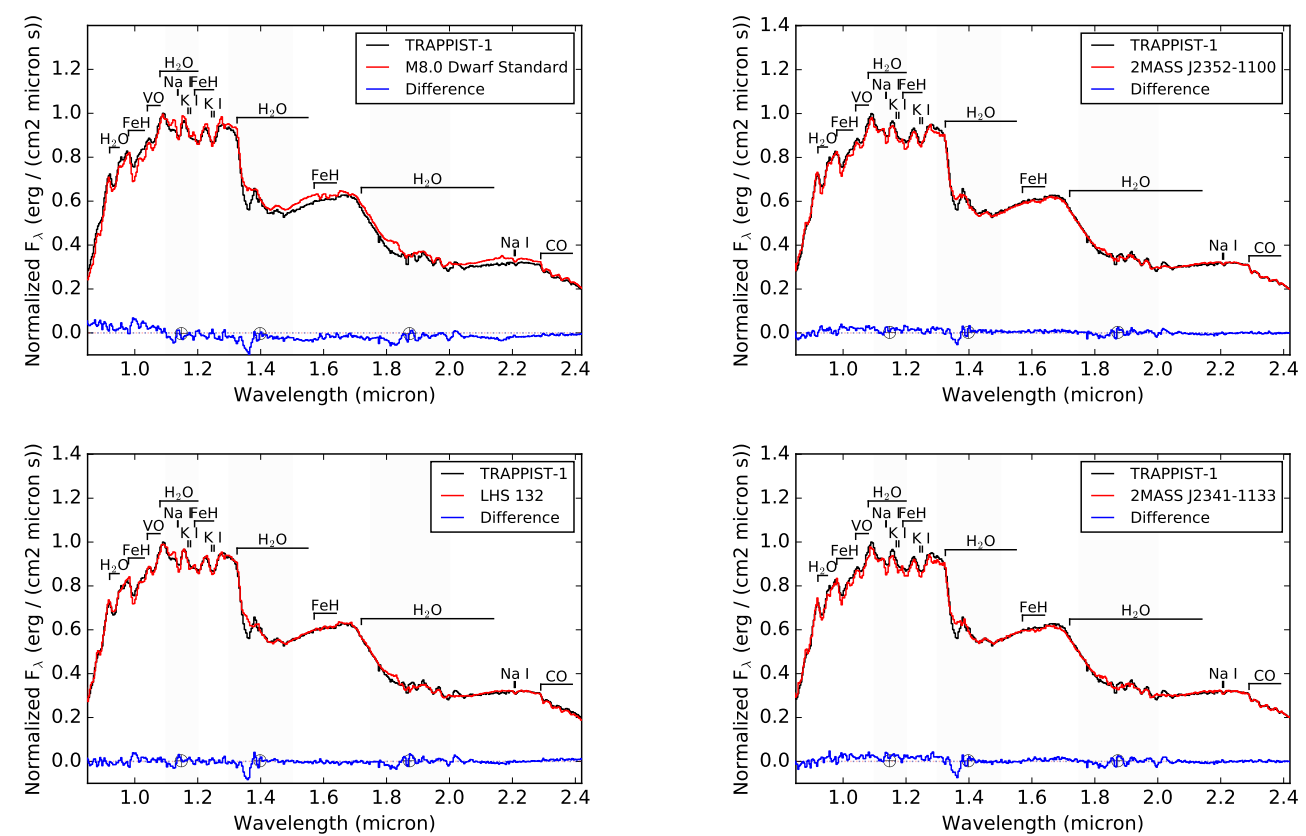

Figure 2. Comparison of the low-resolution near-infrared spectrum of TRAPPIST-1 (Gillon et al. 2016; black line) to equivalent data (red lines) for the M8 standard VB 10 (top left) and the M8 dwarfs 2MASS J2352050-110043 (top right), LHS 132 (bottom left), and 2MASS J2341286-113335 (bottom right). All comparison spectra are from Bardalez Gagliuffi et al. (2014), and are normalized to optimize agreement to the spectrum of TRAPPIST-1 outside telluric absorption bands (grey regions). Difference spectra are shown in blue. Absorption features attributable to $\mathrm{Na}$ I, $\mathrm{K} \mathrm{I}, \mathrm{H}_{2} \mathrm{O}, \mathrm{CO}$, $\mathrm{VO}$ and $\mathrm{FeH}$ are labeled.

Teegarden et al. 2003; Henry et al. 2006; Gagné et al. 2015b) and the d/sdM7 metalpoor companion GJ 660.1B (Aganze et al. 2016). Given the apparent interplay between surface gravity and metallicity effects in index-based gravity metrics for late M dwarfs, we discount the INT-G classification as evidence of youth for TRAPPIST-1.

\subsection{Age Constraints from Metallicity}

While age-metallicity correlations are generally weak among stellar populations, the dispersion of stellar metallicities increases for populations older than a few Gyr, and median metallicity decreases for populations older than $\approx 10$ Gyr (Edvardsson et al. 1993; Haywood et al. 2013; Bergemann et al. 2014). Hence, comparison of TRAPPIST-1's metallicity to population distributions can provide a statistical constraint on its age.

To quantify this diagnostic, we examined the age and metallicity distributions of stars drawn from the Spectroscopic Properties of Cool Stars (SPOCS; Valenti \& Fischer 2005) and an updated analysis of the Geneva-Copenhagen Survey (GCS; Casagrande et al. 2011). In both samples, ages are inferred by comparison of CMDs to model isochrones (for GCS, we used the ages inferred from the Padova isochrones; Bertelli et al. 2008), while metallicities are determined from spectroscopic measurements in SPOCS and Strömgren photometry in GCS. For both samples, we selected $\mathrm{M} \leq 1 \mathrm{M}_{\odot}$ stars with $-0.04<[\mathrm{Fe} / \mathrm{H}]<+0.12$ and parallactic distances within 

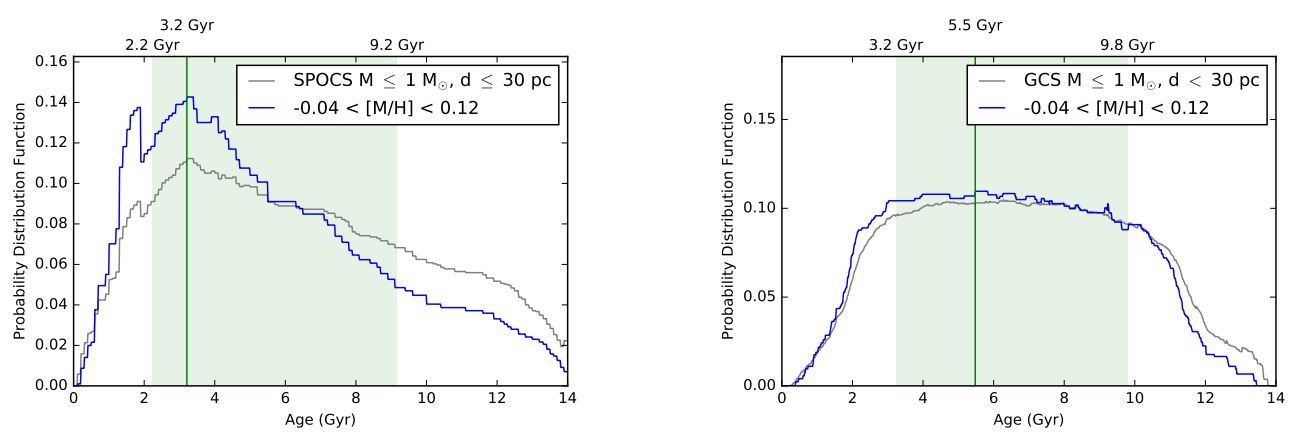

Figure 3. Age probability distribution functions for stars with $\mathrm{M} \leq 1 \mathrm{M}_{\odot},-0.04<$ $[\mathrm{Fe} / \mathrm{H}]<+0.12$ and distances $\leq 30 \mathrm{pc}$ in the SPOCS (left) and GCS (right) catalogs. Grey histograms are without the metallicity constraint; blue histograms are with the metallicity constraint. The solid vertical lines indicate the maximum likelihoods of the age distributions with all constraints and the shaded green regions encompass the $16 \%$ to $84 \%$ probability ranges.

$30 \mathrm{pc}$, and constructed an age distribution by assuming a uniform likelihood for each star between the minimum and maxium ages (SPOCS) or $16 \%$ and $84 \%$ Padova isochronal ages (GCS). These distributions are shown in Figure 3. In both samples, stars younger than 1-2 Gyr and older than 11-12 Gyr are relatively rare. The SPOCS age distribution peaks at young ages, which is enhanced by the metallicity constraint. The GCS age distribution is flat between 2-10 Gyr with a slight preferance toward younger ages with the metallicity constraint. While the maximum likelihood ages are quite different between these samples, their distributions overlap, and we infer ages of 3.2 $2_{-1.0}^{+6.0}$ Gyr for SPOCS and 5.5 $5_{-2.3}^{+3.7}$ Gyr for GCS. As anticipated, the uncertainties are considerable and do little to reduce the overall uncertainty in the system's age.

\subsection{Age Constraints from Kinematics}

Reiners \& Basri (2009) and Burgasser et al. (2015) previously reported equivalent $U V W$ kinematics for TRAPPIST-1 based on radial velocity and proper motion measurements, the latter study concluding that the star is a borderline thin/thick disk star based on the criteria of Bensby et al. (2003). We update this analysis using the more precise radial velocity reported in Barnes et al. (2014) and astrometry reported in Weinberger et al. (2016). The corresponding heliocentric $U V W$ velocities are given in Table 1. Adopting the Local Standard of Rest (LSR) correction of Dehnen \& Binney (1998) used by Bensby et al. (2003), $U V W_{\odot}=[+10.00,+5.25,+7.17]$, we find probabilities of kinematic association $P($ thin $)=81 \%, P($ thick $)=19 \%$ and $P($ halo $)<$ $0.1 \%$. With $P$ (thick $) / P($ thin $)=0.23$, this star remains a borderline thin/thick disk star by the Bensby et al. (2003) criteria.

To derive a quantitative estimate of TRAPPIST-1's kinematic age, we took advantage of the fact that the $V$-velocity asymmetric drift of stellar populations increases over time as the velocity scatter increases $\left(V_{a} \propto \sigma_{U}^{2}\right.$; Strömberg 1924). We again used the GCS sample and examined the age distribution of stars with negative $V$ velocities like TRAPPIST-1. Figure 4 shows the same mass- and distance- constrained distri- 
bution as Figure 3, but with the additional constraint that $V<V_{T 1}=-66 \mathrm{~km} \mathrm{~s}^{-1}$. In this case we see a tilt toward older ages, with a maximum likelihood value of $9.8 \mathrm{Gyr}$, albeit with a wide uncertainty range (3.9-10.5 Gyr). We find a similar distribution (albeit with a very small sample) when a metallicity constraint was also applied to the GCS sample.

As a second approach, we applied a Bayesian method to solve for the probability distribution function of a star's age given its UVW velocities:

$$
P(\text { age } \mid \mathrm{UVW}) \propto P(\mathrm{UVW} \mid \text { age }) P(\text { age })
$$

Here, $P($ age $)$ is the apriori distribution of stellar ages while $P(\mathrm{UVW} \mid$ age $)$ is the distribution of stellar UVW velocities as they evolve over time. We considered three different age priors in our analysis: a constant age distribution (constant star formation rate) up to 12 Gyr; the age distribution of GCS stars with $\mathrm{M} \leq 1 \mathrm{M}_{\odot}$ and $d \leq 30 \mathrm{pc}$ without constraints on metallicity or $V$-velocity; and a constant age distribution with an additional "heating" term modeled after Aumer \& Binney (2009) that deweights older populations that spend less time in the immediate Solar Neighborhood,

$$
\ln P \propto-\frac{\Delta \Phi(Z)}{\sigma_{W}(t)^{2}}
$$

Here, $\Delta \Phi(Z)$ is the vertical gravitational potential difference from the mid-plane to a Galactic height $Z$, taken to be $50 \mathrm{pc}$; and $\sigma_{W}(t)$ is the vertical velocity dispersion in $\mathrm{km} \mathrm{s}^{-1}$ over time $t$ in Gyr, calculated as

$$
\sigma_{W}(t)=8.388(t+0.01)^{0.445}
$$

(Aumer \& Binney 2009). For the Galactic potential, we used the four-component disk, halo, and bulge model of Barros et al. (2016) at a Galactic radius of $8 \mathrm{kpc}$. UVW velocities as a function of age were drawn using the age-dispersion relations of Aumer \& Binney (2009), including an asymmetric drift term $V_{a}=-\frac{\sigma_{U}^{2}}{74 \mathrm{~km} \mathrm{~s}^{-1}}$. We drew $10^{7}$ stars at each simulated time step, and computed the fraction of draws as a function of age whose UVW velocities were within $5 \sigma$ of those of TRAPPIST-1.

Figure 4 shows the resulting distribution of stellar ages for our three age priors. All are strongly skewed toward older ages, with maximum likelihood values ranging from 10.2 Gyr (GCS) to 12 Gyr (constant). The GCS and heating model priors produce similar distributions within $11 \mathrm{Gyr}$, the former dropping off rapidly beyond this in accordance with the underlying sample age distribution. Using the median values as best estimates, we infer ages of $9.2_{-2.7}^{+1.1}$ Gyr and $8.7_{-2.9}^{+2.3}$ Gyr for the GCS and heating model priors, which are on the high end but consistent with the other age diagnostics.

\subsection{Age Constraints from Rotation}

While the timescales for rotation spindown and activity decline for ultracool dwarfs become exceedingly long compared to more massive stars (West et al. 2008; Irwin 

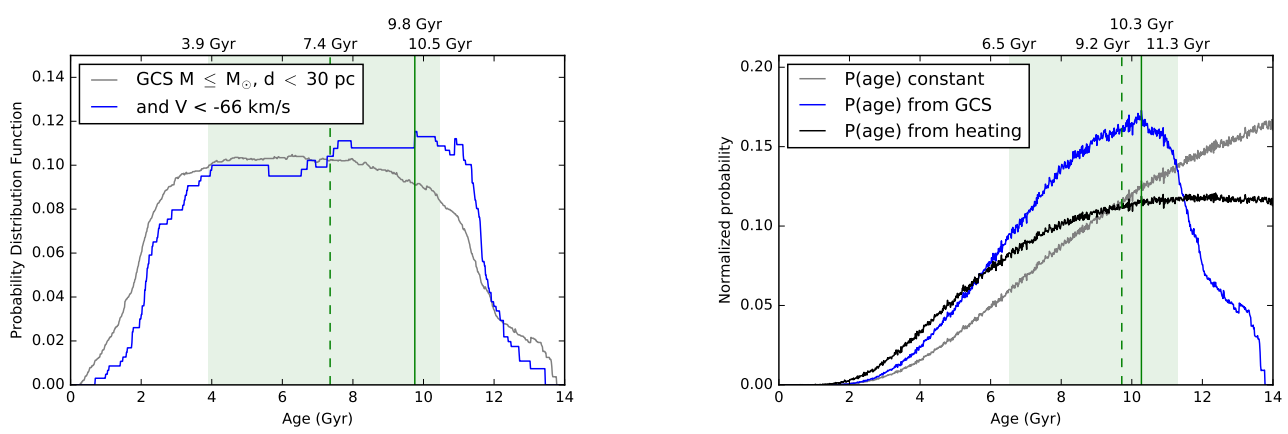

Figure 4. (Left) Distribution of ages of $\mathrm{M}<1 \mathrm{M}_{\odot}$ GCS stars (grey) compared to those with $V<-66 \mathrm{~km} \mathrm{~s}^{-1}$ (blue). (Right) Distribution of ages for a simulated population with $U V W$ dispersions based on three different age priors: a constant prior (grey), a prior based on the GCS sample $\left(\mathrm{M} \leq 1 \mathrm{M}_{\odot}\right.$ and $d \leq 30 \mathrm{pc}$; blue) and a prior based on a model for kinematic heating (black). In both panels, the solid vertical lines indicate the maximum likelihood values of the age distributions for the velocity-selected (left) and GCS prior (right) samples, the dashed vertical lines the medians, and the shaded green regions encompass the $16 \%$ to $84 \%$ probability ranges.

et al. 2011), there is evidence that both properties do evolve in a measureable way (e.g., Burgasser et al. 2015). Early analysis of TRAPPIST-1 has suggested that its 3.295 day rotation period is average for late-type M dwarfs, suggesting a "middle-age" star (3-8 Gyr from Luger et al. 2017).

To quantify this, we compared the rotation period of TRAPPIST-1 to those of midand late-M dwarfs observed through the MEarth program (Nutzman \& Charbonneau 2008) as reported in Newton et al. (2016). Selecting subsamples of stars with significant periodic variability $\left(A / \sigma_{A}>2\right)$ in $0.02 \mathrm{M}_{\odot}$ bins over masses of $0.07-0.18 \mathrm{M}_{\odot}$, we computed the fraction of each subsample that had periods longer than TRAPPIST-1. Figure 5 shows the resulting trend, illustrating that roughly $60 \%$ of the stars observed in this study were slower rotators, suggesting that they are older. If we assume (simplistically) that rotation declines monotonically over time, and use the GCS sample as an age prior, this analysis would suggest an age of $\sim 4-5$ Gyr for TRAPPIST-1, depending on the assumed age of the Milky Way.

However, the rotation periods of very low mass stars at late ages are highly sensitive to initial conditions and the mechanism for angular momentum loss. Figure 5 shows the evolution of rotation period for a $0.08 \mathrm{M}_{\odot}$ star following the angular momentum loss rate prescription of Chaboyer et al. (1995) and Krishnamurthi et al. (1997) as previously applied to low-mass stars (e.g., Bouvier et al. 1997; Reiners \& Basri 2008; Irwin et al. 2011). We assumed a critical (or saturation) rotation rate $\omega_{\text {crit }}$

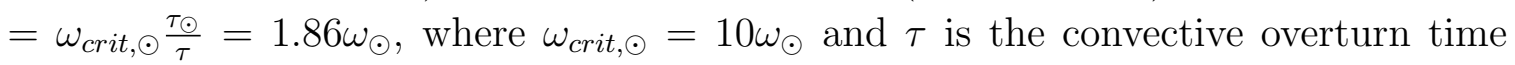
assumed proportional to $\mathrm{M}^{-2 / 3}$ (Reiners \& Basri 2008). We used the time-dependent radii for an $\mathrm{M}=0.08 \mathrm{M}_{\odot}$ from the models of Baraffe et al. (2003). We considered both "slow" and "fast" prescriptions for spindown ${ }^{1}$ from Irwin et al. (2011), as well

\footnotetext{
${ }^{1}$ In the notation of Chaboyer et al. (1995) these are $K_{\text {slow }}=1.20 \times 10^{45} \mathrm{~g} \mathrm{~cm}^{2} \mathrm{~s}=1.25 \times 10^{-10}$ $\mathrm{M}_{\odot} \mathrm{R}_{\odot}^{2} \mathrm{~s}$ and $K_{\text {fast }}=1.12 \times 10^{47} \mathrm{~g} \mathrm{~cm}^{2} \mathrm{~s}=1.16 \times 10^{-8} \mathrm{M}_{\odot} \mathrm{R}_{\odot}^{2} \mathrm{~s}$, respectively.
} 

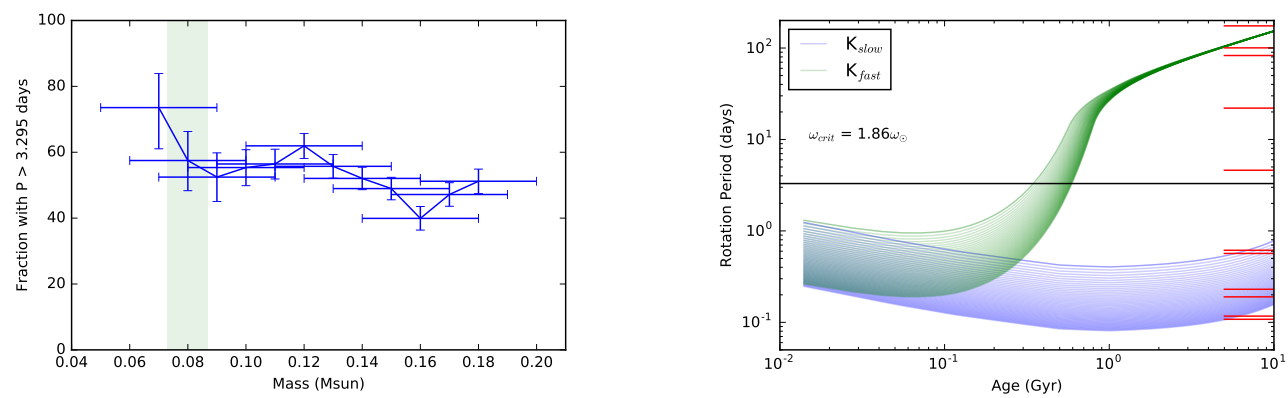

Figure 5. (Left) Fraction of sources in the Newton et al. (2016) sample with rotation periods greater than 3.295 days as a function of mass. Horizontal error bars indicate the sample bin size, vertical error bars the binomial sampling uncertainties. For the mass estimate of TRAPPIST-1, just over half of the sample spins slower, suggesting this source is roughly "middle-age" for an ultracool M dwarf. (Right) Angular momentum evolution for a $0.08 \mathrm{M}_{\odot}$ star based on "slow" (blue lines) and "fast" (green lines) momentum loss following the prescription of Chaboyer et al. (1995) and Irwin et al. (2011). The various lines sample initial rotation rates of $20-100 \omega_{\odot}$. The observed rotation period of TRAPPIST-1 is indicated by the solid black line; the red marks to the right of the panel indicate rotation periods measured for $0.06-0.10 \mathrm{M}_{\odot}$ stars by Newton et al. (2016).

as a range of initial rotation velocities at $\sim 20-50$ Myr of $20-100 \omega_{\odot}$, based on measurements for $\mathrm{M}<0.35 \mathrm{M}_{\odot}$ stars in NGC 2547 reported in the same study. As shown in Figure 5, different angular momentum loss prescriptions produce widely dispersed rotation periods beyond $300 \mathrm{Myr}$, spanning three orders of magnitude by 10 Gyr. This range is well-matched to the range of observed rotation periods for significantly variable $0.06-0.10 \mathrm{M}_{\odot}$ stars in the sample of Newton et al. (2016), which span 0.11-364 days. TRAPPIST-1's rotation period resides between the slow and fast evolutionary tracks. Since neither the specific mechanism of spin-down nor the initial rotation rate are known for this source, at best we can conclude that TRAPPIST-1 is likely older than 300 Myr, the age at which the "fast" track periods exceed 3.3 days.

\subsection{Age Constraints from Activity}

Low-mass stars show clear age-activity correlations related to the spindown of stars and reduction of rotationally-driven magnetic dynamos (Skumanich 1972; Feigelson \& Lawson 2004; Covey et al. 2008). As spindown timescales increase for the lowestmass stars, saturated magnetic emission can persist for even slowly rotating stars ( $P<86$ day) with little correlation between the incidence of emission and rotation period (West et al. 2008, 2015). However, there is evidence for a correlation between the strength of $\mathrm{H} \alpha$ emission and "stratigraphic" age (distance from the Galactic plane) that continues through the end of the M dwarf sequence.

Persistent $\mathrm{H} \alpha$ emission from TRAPPIST-1 is weaker than emission in over half of the active late-M dwarfs near the Sun (Figure 6), suggesting an age in the upper half of this sample. This comparison stands in contrast to claims that TRAPPIST-1 is "highly active", suggesting youth (e.g., Bourrier et al. 2017; Vida et al. 2017). The perception that TRAPPIST-1 is highly active is also related to its flaring emission, 

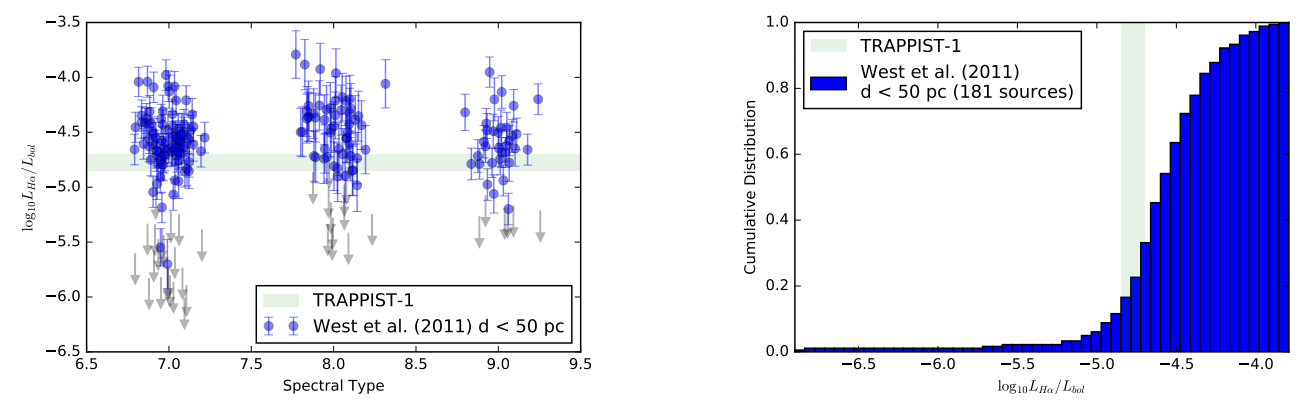

Figure 6. (Left) Measurements of $\log _{10} \mathrm{~L}_{\mathrm{H} \alpha} / \mathrm{L}_{\text {bol }}$ for 239 M7-M9 dwarfs with 50 pc with SDSS spectra as reported by West et al. (2011, circles and arrows) compared to the range of values reported for TRAPPIST-1 (green region; Table 1). Circles with error bars are sources with significant detections ( $\mathrm{H} \alpha$ emission peak more than three times the continuum noise); downward arrows are upper limits. Points are randomly offset along the x-axis to aid in visualization. (Right) Cumulative distribution of significant $\log _{10} \mathrm{~L}_{\mathrm{H} \alpha} / \mathrm{L}_{\mathrm{bol}}$ measurements for the SDSS sample, again compared to the range of measurements for TRAPPIST-1. This star is less active than over half of the nearby M7-M9 dwarfs.

and specifically the detection of a super-solar flare in its $K 2$ lightcurve, with an integrated energy $\mathrm{E}=10^{33} \mathrm{erg}$ (Vida et al. 2017). Again, context is essential. M dwarfs typically have higher optical and infrared flare rates and flare energies than solar-type stars (Davenport et al. 2012) and TRAPPIST-1's flare duty cycle during the $K_{2}$ monitoring period, $\approx 0.1 \%^{2}$ as reported by Vida et al. (2017), is more than an order of magnitude below the $3 \pm 1 \%$ inferred for M7-M9 dwarfs by Hilton et al. (2010). The cumulative flare frequency distribution for TRAPPIST-1 as a function of energy, also reported in Vida et al. (2017), is similarly depressed by a factor of $\approx 4$ compared to other M6-M8 dwarfs (Hilton 2011; Gizis et al. 2017a). Finally, the K2 flare, while dramatic, is not unique; Gizis et al. (2017b) report an E $>4 \times 10^{33}$ erg flare from an L0 dwarf observed with K2 and Schmidt et al. (2014, 2016) have reported E $>10^{34}$ erg flares from M8 and L1 dwarfs detected in the All-Sky Automated Survey for Supernovae survey (Shappee et al. 2014).

Taken together, these activity metrics suggest TRAPPIST-1 is older than the typical late-M dwarf in the Solar Neighborhood, but remains an active star. Given the lack of empirical calibrations for age/activity among the latest $\mathrm{M}$ dwarfs, we are unable to more specifically quantify TRAPPIST-1's age from these measures.

\section{DISCUSSION}

Combining our age probability distribution functions from metallicity and kinematics, and lower limits from the absence of lithium absorption and measured rotation period, we deduce a concordance age of 7.6 \pm 2.2 Gyr for TRAPPIST-1 (Figure 7). This is inconsistent with some of the qualitative estimates reported in the literature (e.g., "relatively young"; Bourrier et al. 2017; “young", O’Malley-James \& Kalteneg-

\footnotetext{
${ }^{2}$ Based on a typical flare time scale of $1-2 \mathrm{~min}=0.02-0.03 \mathrm{hr}$ and the median time between flares $28.1 \mathrm{hr}$.
} 
ger 2017), and is on the high end of the 3-8 Gyr age adopted by Luger et al. (2017). This older age has important implications on both the stability and habitability of its orbiting planets.

In terms of stability, N-body simulations presented in Gillon et al. (2017) showed the planetary system to be consistently unstable on timescales $<0.5 \mathrm{Myr}$, with only an $8 \%$ change of surviving $1 \mathrm{Gyr}$. This is refuted by the much older age we infer for the TRAPPIST-1 star. However, recent simulations show that the resonant configuration of these planets is in fact highly stable through disk migration on timescales of $50 \mathrm{Myr}$ $\left(10^{10}\right.$ orbits), with or without eccentricity dampening. That this system appears to have persisted for over 5 Gyr, despite dynamial interactions that are readily detectable through transit timing variations (Gillon et al. 2017; Wang et al. 2017), suggests that the resonant configuration is indeed inherently stable.

In terms of habitability, despite TRAPPIST-1's modest emission as compared to other late-M dwarfs, the radiation and particle environment is still extreme as compared to the Earth (Bolmont et al. 2017; Wheatley et al. 2017; Garraffo et al. 2017). Based on current estimates of XUV-driven mass loss, the high energy emission of TRAPPIST-1 is likely sufficient to have evaporated an Earth's ocean of water mass from each of the TRAPPIST-1 planets except $g$ and $h$ over the system's lifetime (Bolmont et al. 2017; Bourrier et al. 2017). Moreover, the stripping of atmospheres and oceans may be enhanced by direct interaction between stellar and planetary magnetic field lines, which could funnel stellar wind particles directly to the planets' surfaces (Garraffo et al. 2016, 2017). On the other hand, current estimates of the planets' densities are generally below Earth's average density (Gillon et al. 2017; Wang et al. 2017), suggesting volatile-rich worlds that may have ample reservoirs; while ocean evaporation and hydrogen loss could result in an oxygen- and ozone-rich atmosphere that could shield the surface from high UV fluxes (Luger \& Barnes 2015; O'MalleyJames \& Kaltenegger 2017). Transit spectroscopy measurements of the atmospheres of these planets are currently insufficient to detect the signatures of all but the lightest elements (de Wit et al. 2016), but the James Webb Space Telescope should have the sensitivity to detect Earth-like atmospheres around these plants, if they exist (Barstow \& Irwin 2016).

Finally, we note that agreement between the observed luminosity, average stellar density and evolutionary models can be achieved if the star's radius is modestly inflated relative to model predictions. Our analysis indicates that a radius of $0.121 \pm 0.003 \mathrm{R}_{\odot}$ is needed to bring both Burrows et al. $(1997,2001)$ and Baraffe et al. (2015) evolutionary models in line with the observed properties of TRAPPIST1. This radius is formally consistent with the value adopted in Gillon et al. (2016), and represents a modest $3 \%$ increase in planetary radii and $11 \%$ decrease in inferred planetary densities, less than current uncertainties (Gillon et al. 2017; Wang et al. 2017). 


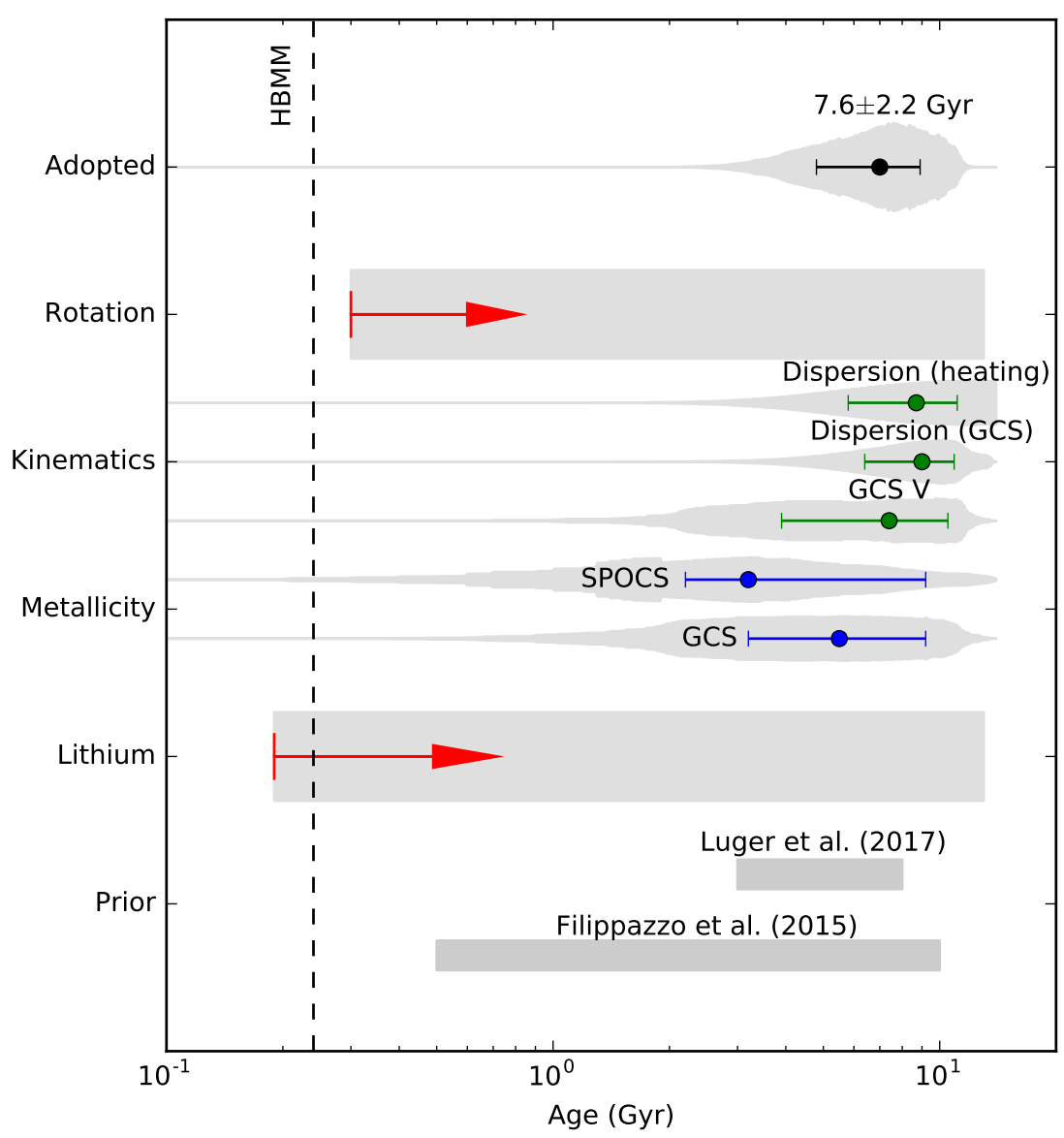

Figure 7. Summary of age estimates for TRAPPIST-1, from bottom to top: original age range from Filippazzo et al. (2015); lower limit based on absence of Li I absorption; age probability distribution functions for GCS and SPOCS stars with similar metallicities; age probability distribution function for GCS stars with $V \leq V_{T 1}=-66 \mathrm{~km} \mathrm{~s}^{-1}$; kinematic dispersion simulations with age priors based on the GCS sample (lower) and heating losses (upper); lower limit based on rotation period; and our concordance age estimate. Throughout, symbols with error bars indicate the maximum likelihood and 16-84\% probability ranges, while the grey shaded regions map the underlying probability distribution functions.

The authors acknolwedge discussions with Eric Agol, Vincent Bourrier, Amaury Triaud, and Valerie van Grootel that aided in the preparation of the manuscript; and thank the Hon. John Culberson of Texas's 7th congressional district, US House of Representatives, for asking about the age of TRAPPIST-1 during his visit to JPL in February 2017, which spurred the writing of this paper. EEM acknowledges the NASA NExSS program for support. AJB acknowledges funding support from the National Science Foundation under award No. AST-1517177. Part of this research was carried out at the Jet Propulsion Laboratory, California Institute of Technology, 
under a contract with the National Aeronautics and Space Administration. This document does not contain export controlled information (URS266250). This material is based upon work supported by the National Aeronautics and Space Administration under Grant No. NNX16AF47G issued through the Astrophysics Data Analysis Program This research has made use of the SIMBAD database, operated at CDS, Strasbourg, France; NASA's Astrophysics Data System Bibliographic Services; the M, L, T, and Y dwarf compendium housed at http://DwarfArchives.org; and the Spex Prism Libraries at http://www. browndwarfs.org/spexprism.

Software: astropy (Astropy Collaboration et al. 2013), SPLAT (Burgasser 2014)

\section{REFERENCES}

Aganze, C., Burgasser, A. J., Faherty, J. K., et al. 2016, AJ, 151, 46

Allen, P. R. 2007, ApJ, 668, 492

Aller, K. M., Liu, M. C., Magnier, E. A., et al. 2016, ApJ, 821, 120

Allers, K. N., \& Liu, M. C. 2013, ApJ, 772,79

Astropy Collaboration, Robitaille, T. P., Tollerud, E. J., et al. 2013, A\&A, 558, A33

Aumer, M., \& Binney, J. J. 2009, MNRAS, 397, 1286

Baraffe, I., Chabrier, G., Barman, T. S., Allard, F., \& Hauschildt, P. H. 2003, A\&A, 402, 701

Baraffe, I., Homeier, D., Allard, F., \& Chabrier, G. 2015, A\&A, 577, A42

Bardalez Gagliuffi, D. C., Burgasser, A. J., Gelino, C. R., et al. 2014, ApJ, 794, 143

Barenfeld, S. A., Bubar, E. J., Mamajek, E. E., \& Young, P. A. 2013, ApJ, 766, 6

Barnes, J. R., Jenkins, J. S., Jones, H. R. A., et al. 2014, MNRAS, 439, 3094

Barros, D. A., Lépine, J. R. D., \& Dias, W. S. 2016, A\&A, 593, A108

Barstow, J. K., \& Irwin, P. G. J. 2016, MNRAS, 461, L92

Bensby, T., Feltzing, S., \& Lundström, I. 2003, A\&A, 410, 527

Bergemann, M., Ruchti, G. R., Serenelli, A., et al. 2014, A\&A, 565, A89
Bertelli, G., Girardi, L., Marigo, P., \& Nasi, E. 2008, A\&A, 484, 815

Bildsten, L., Brown, E. F., Matzner, C. D., \& Ushomirsky, G. 1997, ApJ, 482, 442

Bolmont, E., Selsis, F., Owen, J. E., et al. 2017, MNRAS, 464, 3728

Bourrier, V., Ehrenreich, D., Wheatley, P. J., et al. 2017, A\&A, 599, L3

Bouvier, J., Forestini, M., \& Allain, S. 1997, A\&A, 326, 1023

Bouy, H., Brandner, W., Martín, E. L., et al. 2003, AJ, 126, 1526

Burgasser, A. J. 2014, in Astronomical Society of India Conference Series, Vol. 11, Astronomical Society of India Conference Series, 7-16

Burgasser, A. J., Logsdon, S. E., Gagné, J., et al. 2015, ApJS, 220, 18

Burke, C. J., Pinsonneault, M. H., \& Sills, A. 2004, ApJ, 604, 272

Burrows, A., Hubbard, W. B., Lunine, J. I., \& Liebert, J. 2001, Reviews of Modern Physics, 73, 719

Burrows, A., Marley, M., Hubbard, W. B., et al. 1997, ApJ, 491, 856

Casagrande, L., Schönrich, R., Asplund, M., et al. 2011, A\&A, 530, A138

Chaboyer, B., Demarque, P., \& Pinsonneault, M. H. 1995, ApJ, 441, 865

Chabrier, G., Gallardo, J., \& Baraffe, I. 2007, A\&A, 472, L17 
Covey, K. R., Agüeros, M. A., Green, P. J., et al. 2008, ApJS, 178, 339

Cruz, K. L., Kirkpatrick, J. D., \& Burgasser, A. J. 2009, AJ, 137, 3345

Cruz, K. L., Reid, I. N., Kirkpatrick, J. D., et al. 2007, AJ, 133, 439

Cutri, R. M., Wright, E. L., Conrow, T., et al. 2013, Explanatory Supplement to the AllWISE Data Release Products, Tech. rep.

Davenport, J. R. A., Becker, A. C., Kowalski, A. F., et al. 2012, ApJ, 748, 58

de Wit, J., Wakeford, H. R., Gillon, M., et al. 2016, Nature, 537, 69

Dehnen, W., \& Binney, J. J. 1998, MNRAS, 298, 387

Dieterich, S. B., Henry, T. J., Jao, W.-C., et al. 2014, AJ, 147, 94

Edvardsson, B., Andersen, J., Gustafsson, B., et al. 1993, A\&A, 275, 101

Feigelson, E. D., \& Lawson, W. A. 2004, ApJ, 614, 267

Filippazzo, J. C., Rice, E. L., Faherty, J., et al. 2015, ApJ, 810, 158

Gagné, J., Lafrenière, D., Doyon, R., Malo, L., \& Artigau, É. 2014, ApJ, 783, 121

-. 2015a, ApJ, 798, 73

Gagné, J., Faherty, J. K., Cruz, K. L., et al. 2015b, ApJS, 219, 33

Garraffo, C., Drake, J. J., \& Cohen, O. 2016, ApJL, 833, L4

Garraffo, C., Drake, J. J., Cohen, O., Alvarado-Gomez, J. D., \& Moschou, S. P. 2017, in Radio Exploration of Planetary Habitability (AASTCS5), Vol. 49, 101.06

Gillon, M., Jehin, E., Lederer, S. M., et al. 2016, Nature, 533, 221

Gillon, M., Triaud, A. H. M. J., Demory, B.-O., et al. 2017, Nature, 542, 456

Gizis, J. E., Monet, D. G., Reid, I. N., et al. 2000, AJ, 120, 1085

Gizis, J. E., Paudel, R. R., Mullan, D., et al. 2017a, ArXiv e-prints, arXiv: 1703.08745

Gizis, J. E., Paudel, R. R., Schmidt, S. J., Williams, P. K. G., \& Burgasser, A. J. 2017b, ApJ, 838, 22
Gizis, J. E., Reid, I. N., Knapp, G. R., et al. 2003, AJ, 125, 3302

Haywood, M., Di Matteo, P., Lehnert, M. D., Katz, D., \& Gómez, A. 2013, A\&A, 560, A109

Henry, T. J., Jao, W.-C., Subasavage, J. P., et al. 2006, AJ, 132, 2360

Henry, T. J., Subasavage, J. P., Brown, M. A., et al. 2004, AJ, 128, 2460

Henry, T. J., Walkowicz, L. M., Barto, T. C., \& Golimowski, D. A. 2002, AJ, 123, 2002

Hilton, E. J. 2011, PhD thesis, University of Washington

Hilton, E. J., West, A. A., Hawley, S. L., \& Kowalski, A. F. 2010, AJ, 140, 1402

Irwin, J., Berta, Z. K., Burke, C. J., et al. 2011, ApJ, 727, 56

Janson, M., Hormuth, F., Bergfors, C., et al. 2012, ApJ, 754, 44

Kirkpatrick, J. D., Beichman, C. A., \& Skrutskie, M. F. 1997, ApJ, 476, 311

Kirkpatrick, J. D., Henry, T. J., \& McCarthy, Jr., D. W. 1991, ApJS, 77, 417

Kirkpatrick, J. D., Cruz, K. L., Barman, T. S., et al. 2008, ApJ, 689, 1295

Kirkpatrick, J. D., Looper, D. L., Burgasser, A. J., et al. 2010, ApJS, 190, 100

Kraus, A. L., \& Hillenbrand, L. A. 2012, ApJ, 757, 141

Krishnamurthi, A., Pinsonneault, M. H., Barnes, S., \& Sofia, S. 1997, ApJ, 480, 303

Leggett, S. K. 1992, ApJS, 82, 351

Liebert, J., \& Gizis, J. E. 2006, PASP, 118,659

López-Morales, M. 2007, ApJ, 660, 732

Luger, R., \& Barnes, R. 2015, Astrobiology, 15, 119

Luger, R., Sestovic, M., Kruse, E., et al. 2017, ArXiv e-prints, arXiv:1703.04166

Luhman, K. L., Stauffer, J. R., \& Mamajek, E. E. 2005, ApJL, 628, L69

MacDonald, J., \& Mullan, D. J. 2009, ApJ, 700, 387

Malo, L., Doyon, R., Lafrenière, D., et al. 2013, ApJ, 762, 88 
Mann, A. W., Feiden, G. A., Gaidos, E., Boyajian, T., \& von Braun, K. 2015, ApJ, 804, 64

Mohanty, S., Stassun, K. G., \&

Doppmann, G. W. 2010, ApJ, 722, 1138

Newton, E. R., Charbonneau, D., Irwin, J., et al. 2014, AJ, 147, 20

Newton, E. R., Irwin, J., Charbonneau, D., et al. 2016, ApJ, 821, 93

Nutzman, P., \& Charbonneau, D. 2008, PASP, 120, 317

O'Malley-James, J. T., \& Kaltenegger, L. 2017, MNRAS, 469, L26

Reiners, A., \& Basri, G. 2008, ApJ, 684, 1390

—. 2009, ApJ, 705, 1416

Reiners, A., Seifahrt, A., Stassun, K. G., Melo, C., \& Mathieu, R. D. 2007, ApJL, 671, L149

Schmidt, S. J., Cruz, K. L., Bongiorno, B. J., Liebert, J., \& Reid, I. N. 2007, AJ, 133, 2258

Schmidt, S. J., Prieto, J. L., Stanek, K. Z., et al. 2014, ApJL, 781, L24

Schmidt, S. J., Shappee, B. J., Gagné, J., et al. 2016, ApJL, 828, L22

Seager, S., \& Mallén-Ornelas, G. 2003, ApJ, 585, 1038

Sestito, P., \& Randich, S. 2005, A\&A, 442, 615

Shappee, B. J., Prieto, J. L., Grupe, D., et al. 2014, ApJ, 788, 48

Siegler, N., Close, L. M., Mamajek, E. E., \& Freed, M. 2003, ApJ, 598, 1265

Skrutskie, M. F., Cutri, R. M., Stiening, R., et al. 2006, AJ, 131, 1163
Skumanich, A. 1972, ApJ, 171, 565

Soderblom, D. R. 2010, ARA\&A, 48, 581

Stassun, K. G., Kratter, K. M., Scholz, A., \& Dupuy, T. J. 2012, ApJ, 756, 47

Stauffer, J. R., Schultz, G., \& Kirkpatrick, J. D. 1998, ApJL, 499, L199+

Strömberg, G. 1924, ApJ, 59, 228

Tanner, A., White, R., Bailey, J., et al. 2012, ApJS, 203, 10

Teegarden, B. J., Pravdo, S. H., Hicks, M., et al. 2003, ApJL, 589, L51

Valenti, J. A., \& Fischer, D. A. 2005, ApJS, 159, 141

Vida, K., Kővári, Z., Pál, A., Oláh, K., \& Kriskovics, L. 2017, ArXiv e-prints, arXiv:1703.10130

Wang, S., Wu, D.-H., Barclay, T., \& Laughlin, G. P. 2017, ArXiv e-prints, arXiv:1704.04290

Weinberger, A. J., Boss, A. P., Keiser, S. A., et al. 2016, AJ, 152, 24

West, A. A., Hawley, S. L., Bochanski, J. J., et al. 2008, AJ, 135, 785

West, A. A., Weisenburger, K. L., Irwin, J., et al. 2015, ApJ, 812, 3

West, A. A., Morgan, D. P., Bochanski, J. J., et al. 2011, AJ, 141, 97

Wheatley, P. J., Louden, T., Bourrier, V., Ehrenreich, D., \& Gillon, M. 2017, MNRAS, 465, L74

Winters, J. G., Henry, T. J., Lurie, J. C., et al. 2015, AJ, 149, 5 